
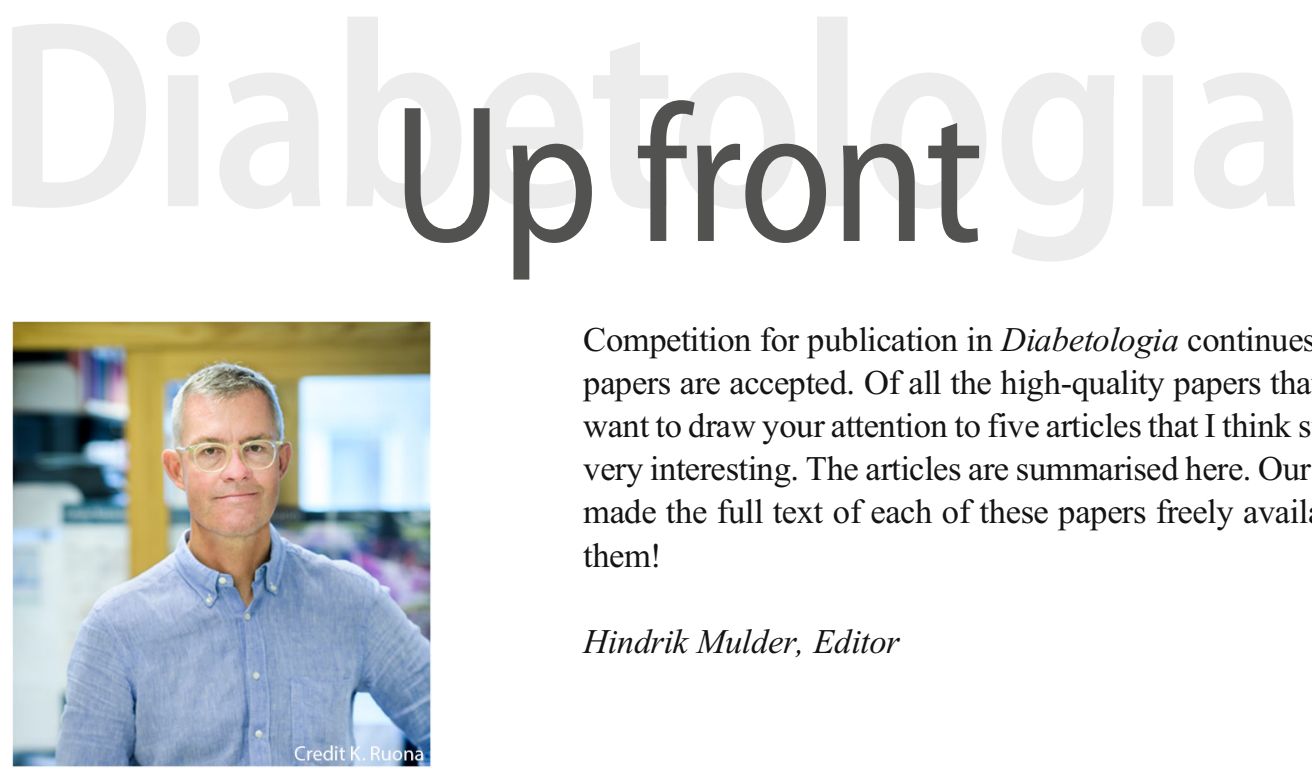

Competition for publication in Diabetologia continues to grow, and less than $20 \%$ of papers are accepted. Of all the high-quality papers that appear in this month's issue I want to draw your attention to five articles that I think stand out in some regard and are very interesting. The articles are summarised here. Our publisher, Springer, has kindly made the full text of each of these papers freely available. I hope you enjoy reading them!

Hindrik Mulder, Editor

\section{Brain JNK and metabolic disease}

Rubén Nogueiras, Guadalupe Sabio

Obesity has become a global epidemic. In recent years, major efforts have been made to define the role played by the central nervous system (CNS) in this disease. Several CNS circuits are altered in obese individuals, and these changes can induce uncontrolled hunger and/or decreased energy expenditure, the latter being associated with physical inactivity, reduced baseline metabolism and poor thermogenic capacity. c-Jun N-terminal kinases (JNKs) in the hypothalamus are among the signalling molecules activated during obesity that may contribute to deleterious molecular adaptations. In this issue, Nogueiras and Sabio (https://doi. org/10.1007/s00125-020-05327-w) review recent findings that reveal opposing pro- and anti-obesogenic actions of JNK family members in the CNS - more precisely the hypothalamus - with regard to the control of food intake and energy expenditure. The authors also discuss the future potential of these kinases as targets in the treatment of obesity.

(D) The figures from this review are available as a downloadable slideset

Impact of age at type 2 diabetes mellitus diagnosis on mortality and vascular complications: systematic review and meta-analyses

$\overline{\text { Natalie Nanayakkara, Andrea J. Curtis, Stephane Heritier, }}$ Adelle M. Gadowski, Meda E. Pavkov, Timothy Kenealy, David R. Owens, Rebecca L. Thomas, Soon Song, Jencia Wong, Juliana C.-N. Chan, Andrea O.-Y. Luk, Giuseppe Penno, Linong Ji, Viswanathan Mohan, Anandakumar
Amutha, Pedro Romero-Aroca, Danijela Gasevic, Dianna J. Magliano, Helena J. Teede, John Chalmers, Sophia Zoungas

Type 2 diabetes is increasingly diagnosed in younger adults; however, the relationship between age at diagnosis and diabetes complications is unclear. In this issue, Nanayakkara et al (https:// doi.org/10.1007/s00125-020-05319-w) integrate data from 1.3 million individuals from 30 countries to conduct the first systematic review and meta-analyses exploring associations between age at diabetes diagnosis and subsequent complications. Each 1 year increase in age at diabetes diagnosis was associated with a $4 \%, 3 \%$ and $5 \%$ decreased risk of all-cause mortality, macrovascular disease and microvascular disease, respectively, adjusted for current age. The authors propose that, over time, the effects of both ageing and diabetes duration may be compounded, resulting in premature complications and death in people diagnosed with type 2 diabetes at a younger age. Early and sustained interventions to optimise glycaemic control and cardiovascular risk profiles in those with established type 2 diabetes, and measures to delay the onset of type 2 diabetes in high-risk groups are crucial to reduce future diabetes-related morbidity and mortality.

The impact of hospital-diagnosed depression or use of antidepressants on treatment initiation, adherence and $\mathrm{HbA}_{1 \mathrm{c}}$ LDL target achievement in newly diagnosed type 2 diabetes

Christopher Rohde, Jakob S. Knudsen, Norbert Schmitz, Søren Dinesen Østergaard, Reimar W. Thomsen

Incident depression in type 2 diabetes is associated with poor self-care and suboptimal treatment adherence. However, it is 
not known how pre-existing depression treatment relates to quality of care for individuals with newly diagnosed type 2 diabetes. In this issue, Rohde et al (https://doi.org/10.1007/ s00125-020-05303-4) report that individuals with newly diagnosed type 2 diabetes who already receive treatment for depression are more likely than others to initiate and be adherent to glucose-lowering and lipid-modifying treatment. Pre-existing depression treatment was also associated with successful attainment of LDL-cholesterol and $\mathrm{HbA}_{1 \mathrm{c}}$ treatment targets among individuals with type 2 diabetes. The authors suggest that these findings may be driven by the fact that individuals with ongoing depression treatment are already familiar with the healthcare system and drug treatment, and that effective treatment of depression, in itself, may improve diabetes care.

Increased particle size of triacylglycerol-enriched remnant lipoproteins, but not their plasma concentration or lipid content, augments risk prediction of incident type 2 diabetes

Luiz Sérgio F. Carvalho, Isabela M. Benseñor, Ana C. C. Nogueira, Bruce B. Duncan, Maria I. Schmidt, Michael J. Blaha, Peter P. Toth, Steven R. Jones, Raul D. Santos, Paulo A. Lotufo, Andrei C. Sposito, on behalf of the ELSA-Brasil study

Little is known regarding risk prediction of type 2 diabetes before the detection of glucose abnormalities; however, up to $3 \%$ of individuals in the general population who are not in the impaired fasting glucose range develop diabetes each year. In this issue, Carvalho et al (https://doi.org/10.1007/s00125-02005322-1) hypothesise that, among the pathophysiological processes that play a role in type 2 diabetes, the loading of triacylglycerol-enriched remnant lipoproteins (TRLs) with lipids (resulting in large particles) could be useful for risk prediction. The authors report that, when included in a risk prediction model, TRL particle diameter improved the risk prediction of diabetes after 3.7 years of follow-up as compared with a model that did not include this variable, particularly in individuals with normal $\mathrm{HbA}_{1 \mathrm{c}}$ levels at baseline. These data support TRL particle size as a risk factor that is changed early in the pathophysiological processes that lead to the development of type 2 diabetes, even before glucose abnormalities are established.

Effects of long-term vitamin $D$ and $n-3$ fatty acid supplementation on inflammatory and cardiac biomarkers in patients with type 2 diabetes: secondary analyses from a randomised controlled trial

Christine P. Limonte, Leila R. Zelnick, John Ruzinski, Andrew N. Hoofnagle, Ravi Thadhani, Michal L. Melamed, I-Min Lee, Julie E. Buring, Howard D. Sesso, JoAnn E. Manson, Ian H. de Boer

Immune system activation contributes to the development of diabetes complications. Vitamin $\mathrm{D}$ and $n-3$ fatty acids have been recognised for their anti-inflammatory effects in laboratory models, with mixed evidence from trials in diabetes populations. In this issue, Limonte et al (https://doi.org/10.1007/ s00125-020-05300-7) report results of changes in serum concentrations of inflammatory and cardiovascular biomarkers from the Vitamin D and Omega-3 Trial to Prevent and Treat Diabetic Kidney Disease (VITAL-DKD), in which 1312 adults with type 2 diabetes were randomised to supplementation with vitamin $\mathrm{D}_{3}$ (2000 IU/day) and/or $n-3$ fatty acids (eicosapentaenoic acid and docosahexaenoic acid; $1 \mathrm{~g} /$ day) or placebo and followed for 5 years. The authors found that supplementation did not lead to a decline in serum levels of IL-6, high-sensitivity C-reactive protein or $\mathrm{N}$ terminal pro-B-type natriuretic peptide. They conclude that this study tilts the balance of available evidence to favour no benefit of these interventions on inflammation and suggests that supplementation is unlikely to have a positive impact on diabetes complications for which inflammation is an underlying cause.

All text supplied by the authors.

Publisher's note Springer Nature remains neutral with regard to jurisdictional claims in published maps and institutional affiliations. 